\title{
Acute blood pressure response in hypertensive elderly women immediately after water aerobics exercise: A crossover study
}

\section{Raphael Martins Cunha, José Vilaça-Alves, Marcelo Vasconcelos Noleto, Juliana Sá Silva, Andressa Moura Costa, Christoffer Novais Farias Silva, Thaís Inácio Rolim Póvoa \& Alexandre Machado Lehnen}

To cite this article: Raphael Martins Cunha, José Vilaça-Alves, Marcelo Vasconcelos Noleto, Juliana Sá Silva, Andressa Moura Costa, Christoffer Novais Farias Silva, Thaís Inácio Rolim Póvoa \& Alexandre Machado Lehnen (2017): Acute blood pressure response in hypertensive elderly women immediately after water aerobics exercise: A crossover study, Clinical and Experimental Hypertension, DOI: 10.1080/10641963.2016.1226891

To link to this article: http://dx.doi.org/10.1080/10641963.2016.1226891

册 Published online: 03 Jan 2017.

Submit your article to this journal ๘

View related articles $\nearrow$

View Crossmark data $\nearrow$ 


\title{
Acute blood pressure response in hypertensive elderly women immediately after water aerobics exercise: A crossover study
}

\author{
Raphael Martins Cunhaa,b, José Vilaça-Alvesc, Marcelo Vasconcelos Noletob, Juliana Sá Silva ${ }^{\mathrm{d}}$, Andressa Moura Costa \\ Christoffer Novais Farias Silva ${ }^{b}$, Thaís Inácio Rolim Póvoab, and Alexandre Machado Lehnen ${ }^{a}$

\begin{abstract}
aExperimental Medicine, Institute of Cardiology of Rio Grande do Sul/University Foundation of Cardiology, Porto Alegre, Rio Grande do Sul, Brazil; ${ }^{b}$ Exercise Physiology Laboratory, State University of Goiás, Goiás, Goiás, Brazil; 'Department of Sport Sciences, Trás-os-Montes e Alto Douro University, Exercise and Health, Vila Real, Portugal; 'Institute of Physical Education, Federal University of Mato Grosso, Cuiabá, Mato Grosso, Brazil
\end{abstract}

\begin{abstract}
Water aerobics exercise is widely recommended for elderly people. However, little is known about the acute effects on hemodynamic variables. Thus, we assessed the effects of a water aerobic session on blood pressure in hypertensive elderly women. Fifty hypertensive elderly women aged $67.8 \pm 4.1$ years, $1.5 \pm 0.6 \mathrm{~m}$ high and BMI $28.6 \pm 3.9 \mathrm{~kg} / \mathrm{m}^{2}$, participated in a crossover clinical trial. The experiment consisted of a 45 -minute water aerobics session (70\%-75\% HRmax adjusted for the aquatic environment) (ES) and a control session (no exercise for 45 minutes) (CS). Heart rate was monitored using a heart rate monitor and systolic blood pressure (SBP) and diastolic (DBP) measurements were taken using a semi-automatic monitor before and immediately after the sessions, and at 10, 20 and 30 minutes thereafter. It was using a generalized estimating equation (GEE) with Bonferroni's post-hoc test ( $p<$ 0.05). At the end of the experimental session, ES showed a rise in SBP of $17.4 \mathrm{mmHg}(14.3 \%, p<0.001)$ and DBP of $5.4 \mathrm{mmHg}(7.8 \%, p<0.001)$ compared to CS. At 10 minutes after exercise, BP declined in ES by a greater magnitude than in CS (SBP $7.5 \mathrm{mmHg}, 6.2 \%, p=0.005$ and DBP $3.8 \mathrm{mmHg}, 5.5 \%, p=0.013$ ). At 20 minutes after exercise and thereafter, SBP and DBP were similar in both ES and CS. In conclusion, $\mathrm{BP}$ returned to control levels within 10-20 minutes remaining unchanged until 30 minutes after exercise, and post-exercise hypotension was not observed. Besides, BP changed after exercise was a safe rise of small magnitude for hypertensive people.
\end{abstract}

\section{ARTICLE HISTORY}

Received 30 April 2016

Revised 15 June 2016

Accepted 15 July 2016

Published online

3 January 2017

\section{KEYWORDS}

Elderly women; exercise training; hypertension; water aerobics

\section{Introduction}

Systemic arterial hypertension is a continuous linear independent risk factor for several cardiovascular conditions and it is associated with a $40 \%$ mortality from stroke and $25 \%$ from coronary artery disease (1). Hypertension is more prevalent among the elderly (2) resulting from aging changes that make individuals more likely to developing it $(3,4)$. In Brazil, the prevalence of hypertension is $68 \%$ among the elderly, which is quite similar to that reported in other countries such as the United States (71\%) (5), Japan (60\%) (6), South Korea (69\%) (7), and Portugal (78\%) (8). Despite its high prevalence, adherence to recommended hypertension treatments is low. In a cohort study of hypertensive patients, $45 \%$ discontinued regular monitoring and only 55\% maintained the desired blood pressure (BP) levels (9).

Regular exercise is a well-established intervention for the prevention and treatment of hypertension (10). Individuals may clinically benefits from post-exercise hypotension (PEH) $(11,12)$ that has longer duration when exercise is performed $2-3$ times per week $(13,14)$. The hypotensive effects of exercise seem to be mainly induced by improved baroreflex sensitivity (15), sympathetic activity suppression (12), improved cardiac output (16), and release of vasodilators (17).
The effects of exercise on land are well documented especially aerobic exercise but evidence of the effects of exercise in water is still scarce. Water aerobics-aerobic exercise in an aquatic environment-has been proposed as a physiologically beneficial exercise modality for the elderly as it can improve cardiorespiratory fitness (18), muscle strength (18), metabolic control, and promote weight reduction (19). Water aerobic exercise is also recommended due to lower risk of injuries as the buoyance of water reduces body weight by $\sim 90 \%$ (20). Most elderly have musculoskeletal conditions and exercise in an aquatic environment can minimize the potential risk of falls (21).

Many studies have investigated exercise and blood pressure response, but almost all studied land exercise (22). Water exercise and land exercise induce different physiological responses. Immersion in water produces changes in hydrostatic pressure that result in redistribution of blood flow especially in the thoracic region and an increase in venous return, stroke volume, and cardiac output (23-25) producing reflex bradycardia (25). Suppression of the renin-angiotensinaldosterone system (26) is also observed. Few studies have investigated water aerobics (22), and showed inconsistent results including increase (24), decrease (27), and even no change (23) in BP levels after exercise.

CONTACT Dr. Alexandre Machado Lehnen amlehnen@gmail.com E Instituto de Cardiologia do Rio Grande do Sul, Av. Princesa Isabel, 395 Santana, $90620-001$ Porto Alegre, RS, Brazil.

(C) 2016 Taylor \& Francis 
Few studies have investigated PEH after water exercise and most of them have examined changes in BP after 30 minutes, 1 hour, and even 24 hours after exercise. A single study was conducted in elderly people. It is thus important to examine potential changes in BP immediately after an aerobic exercise session for hypertensive elderly individuals considering the very properties of water that may affect $\mathrm{BP}$, the effects of physical exertion and a change in the external environment (from in to out of the water). Thus, this study aimed to assess the effects of a water aerobic session on blood pressure in hypertensive elderly women.

\section{Methods}

We conducted a randomized clinical trial with a crossover design. This research project was reviewed and approved by the local institutional review board, and it follows the CONSORT Statement (28) and the principles of the Declaration of Helsinki. All participants read and signed an informed consent form before participating in the study.

\section{Study sample}

The minimum sample size was determined based on a previous study conducted by this research group (29). We considered SBP as the primary endpoint and calculated for a 5\% significance level, power of $80 \%$ and a $10-\mathrm{mmHg}$ difference in SBP. Participants were recruited using local media advertisement. Sixty-three women entered the study, but 13 were excluded from the analyses as they did not complete the entire protocol. The final sample consisted of 50 participants $(n=50)$ - Figure 1 .
The inclusion criteria were being diagnosed with systemic arterial hypertension according to the guidelines of the American Heart Association Seventh Report of the Joint National Committee on Prevention, Detection, Evaluation, and Treatment of High Blood Pressure (1); aged 65-80 years; pre-study $\mathrm{SBP} \leq 160 \mathrm{mmHg}$ and $\mathrm{DBP} \leq 100 \mathrm{mmHg}$, measured at the doctor's office (Table 2); and water aerobic exercise training for at least 6 months. The exclusion criteria were hypereactive individuals $(\mathrm{SBP}>220 \mathrm{mmHg}$ and/or increase in DBP > $150 \mathrm{mmHg}$ during testing); fever and/or any infectious diseases; use of insulin or metformin; Class 2 obesity or higher; heart failure; recent cardiovascular event (in last 3 months); chronic renal failure; active smoking; orthopedic impairments; or physical or mental limitations that prevent exercising.

\section{Study procedures}

On visit 1 to the study site, we explained the participants all the study procedures and answered their questions. Those who agreed to participate were asked to sign a free informed consent form. During this same visit, their medical history was taken and they underwent a physical assessment.

On visit 2, measurements of body weight, height and body mass index (BMI) were taken. The participants underwent randomization: a randomized code was generated (www.ran domization.org) and they were randomly assigned to either the experimental (ES) or the control session (CS) (1:1 allocation ratio) into two groups of $50(\mathrm{n}=50 /$ group $)$ as each participant functioned as their own control.

All sessions were held at the same time $(11 \mathrm{am})$ with 72 hours between sessions. The Figure 1 shows study design.

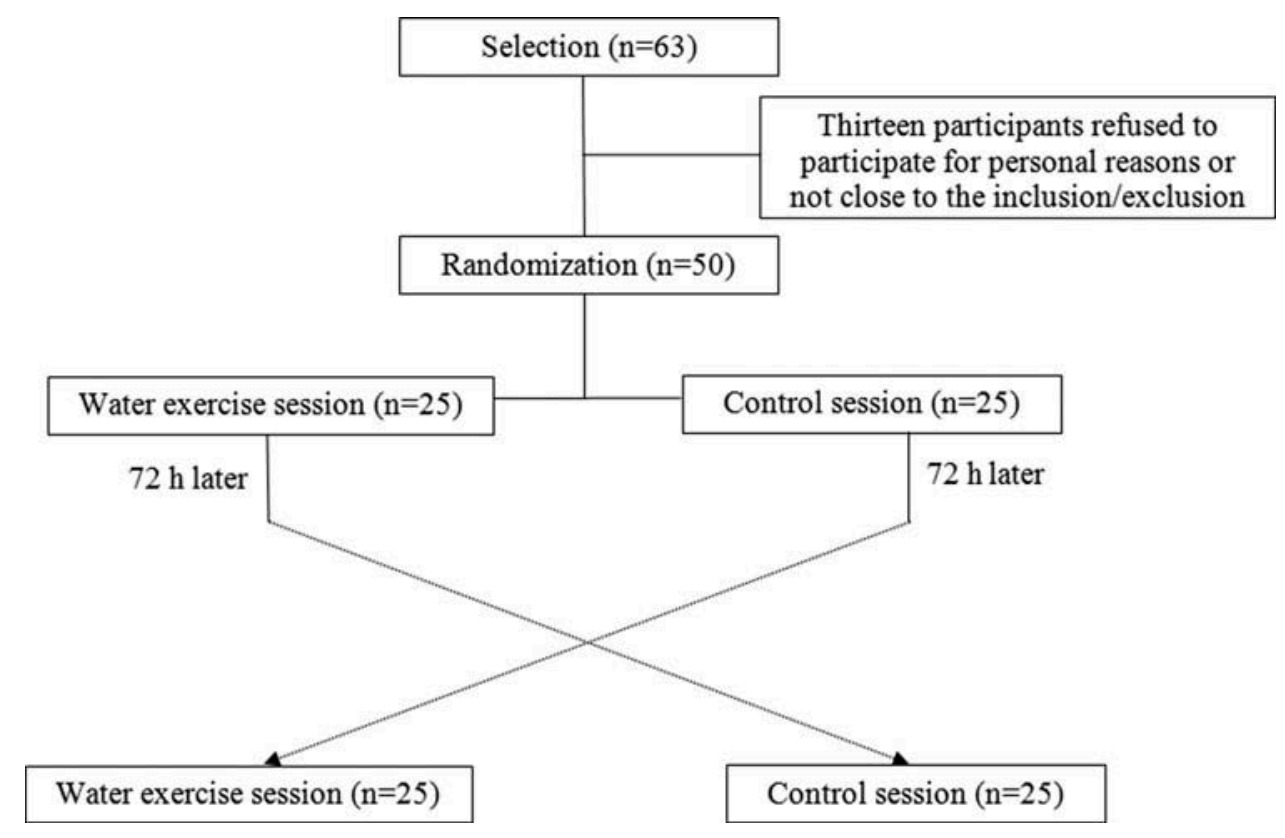

Figure 1. Design of the study. Sixty-three hypertensive women were invited to participate in the study. Thirteen participants refused to participate for personal reasons or did not meet the inclusion/exclusion criteria. Fifty participants were randomized into water exercise or control groups. Seventy-two hours after the first sessions participants took part in the second study session (water exercise or control session). 


\section{Experimental and control sessions}

The experimental session (ES) lasted 45 minutes and consisted of a dynamic warm-up period (5 minutes), an active exercise period (35 minutes), and a cooldown period (5 minutes). It was a continuous session of water aerobic exercises such as flexion, extension, abduction, and adduction of upper and lower limbs. Heart rate (HR) was used to control the intensity of exercise as all participants wore heart monitors (Polar, RS $800 \mathrm{CX}^{\oplus}$, USA). The maximum heart rate (HRmax) was calculated according to the formula proposed by Kruel et al. (30) for exercise in an aquatic environment: $\mathrm{HR}$ for exercise $=\% \mathrm{x}(\mathrm{HR} \max -\Delta \mathrm{HR})$, where $\%$ is the intensity of exercise, HRmax is maximum heart rate (estimated by 220 - age), and $\Delta H R$ is the difference between resting HR out of pool and resting HR in the pool. The exercise intensities were 55\%-60\% HRmax during the warm-up period, $70 \%-75 \%$ HRmax during the active exercise period, and 55\%-60\% HRmax during the cooldown period (Table 1).

The control session (CS) lasted 45 minutes and was held in environment conditions similar to those of the experimental session. During this session, participants remained seated or standing as desired.

\section{Blood pressure measurements}

Blood pressure measurements were taken in a seated position using an internationally validated semi-automatic BP monitor (Omron 705-CP, Matsusaka, Japan) following the techniques as described in the Seventh Report of the Joint National Committee on Prevention, Detection, Evaluation, and Treatment of High Blood Pressure (1). In all sessions (control and experimental), they were taken at all times out of the water: before the study protocol sessions (pre-session), immediately after the sessions (minute 0), and at 10, 20, and 30 minutes after the session (minute 10 , minute 20 , and minute 30 , respectively).

\section{Statistical analysis}

We performed the Shapiro-Wilk test to assess the normality of distribution of numerical data and the Student's $t$-test to compare the main characteristics of the participants. To assess the effects of exercise on BP on two parameters (session and time) and session interaction (session ${ }^{\star}$ time), we used a generalized estimating equation (GEE) followed by Bonferroni's post-hoc test $(p<0.05)$. The effect size of BP changes in ES compared to CS was calculated by Cohen method (31). All data were described as mean \pm standard deviation and the Statistical Package for Social Sciences (SPSS v21) was used for all analyses.

Table 1. Exercise session in the experimental protocol.

\begin{tabular}{lcc}
\hline Session period & Exercise intensity & Heart rate $(\mathrm{bpm})$ \\
\hline Warm-up (5 min) & $55 \%-60 \%$ & $70.7 \pm 2.2-78.3 \pm 2.4$ \\
Active aerobic exercise (35 min) & $70 \%-75 \%$ & $81.1 \pm 3.0-93.5 \pm 2.0$ \\
Cooldown (5 min) & $55 \%-60 \%$ & $70.7 \pm 2.2-78.3 \pm 2.4$ \\
\hline
\end{tabular}

bpm, beats per minute
Table 2. Main characteristics of the study participants $(n=50)$.

\begin{tabular}{|c|c|}
\hline & Mean \pm SD $(95 \% C l)$ \\
\hline Age (years) & $67.80 \pm 4.06(66.64-68.95)$ Min $60.0 ; \max 77.0$ \\
\hline Body weight $(\mathrm{kg})$ & $67.43 \pm 10.18(64.53-70.32)$ Min 51.0; $\max 91.0$ \\
\hline Height $(\mathrm{cm})$ & $1.53 \pm 0.60(1.51-1.55)$ Min $1.4 ; \max 1.6$ \\
\hline $\operatorname{BMI}\left(\mathrm{kg} / \mathrm{m}^{2}\right)$ & $28.62 \pm 3.86(27.52-29.72)$ Min $22.1 ; \max 34.8$ \\
\hline Resting HR (bpm) & $70.90 \pm 11.02(66.71-75.09)$ Min 50.0; $\max 93.0$ \\
\hline Resting SBP $(\mathrm{mmHg})$ & $113.90 \pm 12.26(109.30-118.50)$ Min $96.0 ; \max 145.0$ \\
\hline Resting DBP (mmHg) & $66.40 \pm 9.70(62.78-70.01)$ Min 48.0; $\max 90.0$ \\
\hline
\end{tabular}

\section{Results}

Sixty-three participants entered the study, but 13 were excluded from the analysis as they did not complete the entire protocol, totaling 50 participants $(n=50)$. Table 2 summarizes the main characteristics of the participants. We can see low variance and narrow confidence intervals suggesting homogeneity of the sample. In addition, resting BP measured during sample selection indicates that participants generally had an adequate BP control.

At the beginning of the protocol sessions heart rate was similar in both sessions (CS: $97.1 \pm 8.9$ vs. ES: $93.2 \pm 10.7$ bpm, $p=0.882$ ). The same was seen for SBP (CS: $123.2 \pm 2.4$ vs. ES: $123.9 \pm 2.3 \mathrm{mmHg}, p=0.759$ ) and DBP (CS: $69.9 \pm 1.4$ vs. ES: $69.4 \pm 1.5 \mathrm{mmHg}, p=0.703)--$ Figure 2 .

At the end of the experimental session, there was a rise of $17.4 \mathrm{mmHg}(14.3 \%, p<0.001)$ in SBP as compared with CS (effect size 0.96). Similarly, DBP also increased compared to CS but this rise was of smaller magnitude $5.4 \mathrm{mmHg}(7.8 \%$, $p<0.001$, effect size 0.60). Compared to CS, at 10 minutes after exercise, SBP and DBP declined by $7.5 \mathrm{mmHg}(6.2 \%, p=$ 0.005 , effect size 0.43$)$ and $3.8 \mathrm{mmHg}(5.5 \%, p=0.013$, effect size 0.40 ), respectively. At 20 minutes after exercise, SBP and DBP values were not statistically different in both sessions (SBP, effect size -0.01 and DBP, effect size 0.27), remaining comparable within 30 minutes after the experimental session (SBP, effect size -0.04 and DBP, effect size 0.25).

When $\mathrm{BP}$ values were analyzed within the same session, we found a rise in SBP $(12.4 \%, p<0.001)$ and DBP $(7.3 \%, p<$ 0.001 ) at minute 0 , compared to pre-exercise moment. At 10 minutes after exercise, SBP returned to pre-exercise levels ( $p=$ 0.144 ) and remained stable within 30 minutes after the experimental session. However, DBP remained higher than preexercise levels (pre-exercise: 69.2 vs. $\min 10: 73.1 \mathrm{mmHg}, p$ $=0.011$ ) returning to pre-exercise levels from 10 minutes to the end of the experiment.

\section{Discussion}

Our study focused on changes in BP immediately after a water aerobics session in hypertensive elderly women. SBP and DBP increased in response to water aerobic exercise, but it was a safe rise of small magnitude. Furthermore, BP levels returned to baseline (pre-exercise levels and compared to the control session) within 10-20 minutes after the exercise session. These data further support that PEH remains until 30 minutes after exercise and prescribing this modality/duration/intensity 

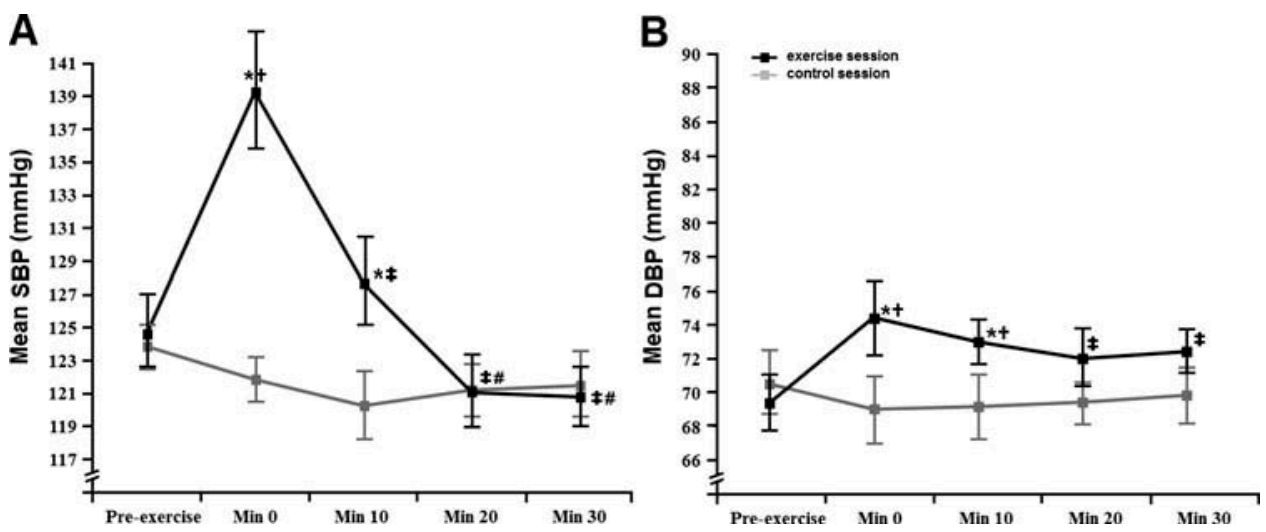

Figure 2. Changes in blood pressure before and after the water aerobic exercise session. Panel $A$, systolic blood pressure, and panel $B$, diastolic blood pressure. ${ }^{*} p<0.05$ vs. control session at same time points, $\uparrow p<0.05$ vs. pre-exercise in the same session, $\neq p<0.05$ vs. minute 0 in the same session, \# vs. minute 10 in the same session.

of exercise is safe for hypertensive elderly individuals (as BP changes were not risky). These findings are consistent with those from other studies conducted by our research group regarding the safety of prescribing for hypertensive elderly women exercise in an aquatic environment (29) and strength exercises (11) and with those from a systematic review by Santos et al. (22). None of these studies reported a marked decline in BP immediately after exercise.

Before the study sessions, BP levels were adequately controlled as recommended in the guidelines (1). BP was controlled with the use of pharmacological anti-hypertensive therapy and physical fitness as non-pharmacological therapy because both contribute to lower BP and reduce cardiovascular risks (1). Physical fitness is associated with chronic effects of exercise and helps control BP. These effects are associated with many forms of exercises $(13,32,33)$ including water aerobics (33), as seen in our sample.

Changes in BP associated with water exercises especially water aerobic exercise may occur due to temperature changes (34), as an individual transitions from water to land. These changes are more significant in elderly people because of their slower metabolism resulting from low lean body mass (35). However, it is noteworthy that, though statistically significant, the rise in BP in our study was within the acceptable range for the participants' age and medical condition (hypertension) (1). It may also be associated with the effects of anti-hypertensive medications taken by the study participants rather than their exercising in water environment. The interaction of anti-hypertensive medication with physical exercise can produce different responses depending on the type of medications used (36-38). Thus, anti-hypertensive medication might have affected BP response immediately after exercise and prevented extremely high BP levels as shown in other studies $(39,40)$.

Given that BP is physiologically a product of cardiac output and peripheral vascular resistance (41) and that in turn cardiac output is determined by the stroke volume and heart rate (41), any change in one or more of these parameters affects BP. The hydrostatic effect of immersion in water redirects $\sim 700 \mathrm{~mL}$ of blood flow from the extremities to the thorax (24), which increases the right intraventricular pressure, stroke volume and cardiac output (23) and decreases heart rate reflexively. Studies have shown a reduction in heart rate during water exercise associated with water depth and temperature. Heithold et al. found heart rate to be 20-29 beats/ min lower during water exercise than land exercise (42). Darby et al. found heart rate to be $7-13$ beats/min lower in $30^{\circ} \mathrm{C}$ water (27). Conversely, when an individual gets out of water after body immersion, a portion of the cardiac output is redistributed to the extremities causing an increase in heart rate and peripheral vascular resistance and thermoregulation (because of the difference between water and environment temperatures) resulting in a rise in BP. Moreover, elderly women climbed up a ladder to get out of the pool. It is an additional physical exertion that might also have contributed to a rise in $\mathrm{BP}$ in the immediate recovery period $(\min 0)$. This may largely explain our finding of a rise in both SBP and DBP at the end of the water aerobic exercise session even after a cooldown period. Indeed, physical exertion during the water aerobic exercise session may by itself explain this rise in BP.

As for environment temperature, CS participants remained fully dressed out of the water and were protected against any potential body heat loss, which does not allow for major comparisons to be made. Heat loss in water occurs through the process of conduction, regulated and controlled by cutaneous vasomotor activity of the vascular network (43). The sympathetic nervous activity increases and promotes vasoconstriction resulting in BP increase (44), which may explain in part $\mathrm{BP}$ responses at least during the early recovery period after water exercise. To maintain the elderly women in the CS in water without exercising would be not feasible in our study because they would lose body heat more rapidly (45).

When we examined BP levels at 10 minutes after exercise, SBP remained higher when compared to CS, but not when compared to baseline (pre-exercise) levels within the same session. This finding can be explained by a slight though not statistically significant decline in SBP in CS at 10 minutes (Figure 2). In turn, DBP remained higher when compared to both CS and pre-exercise levels. Our data at 10 minutes after exercise suggest that the regulatory mechanisms affecting SBP compared to DBP are more sensitive to environmental changes as reported in the literature (46). At 20 and 30 
minutes after exercise, both SBP and DBP returned to baseline levels. Thus, BP levels seem to return to baseline within 10-15 minutes after a moderate-intensity water aerobic exercise session in hypertensive elderly women considering all physiological adjustments of different environments (in and out of water), as previously reported in other studies of our research group (29).

In contrast, we did not observe $\mathrm{PEH}$ during the times studied as reported in other studies $(29,46)$. The hypotensive effect immediately after an exercise session is a safety concern due to the risk of syncope. The phenomenon may be caused by physiological regulation of the cardiovascular system while an individual transitions to a different environment (in and out of the water), and unlike PEH it occurs 30 minutes after exercise. This is corroborated by other studies $(29,46)$ that reported this same effect at 30,60 minutes and 24 hours after exercise.

The results of our study show that water aerobic exercise in well-controlled hypertensive elderly participants was hemodynamically safe and did not bring about an unfavorable rise in BP levels, which is quite significant to this population. However, it should be noted that aerobic exercise training for hypertensive individuals is a first-line non-pharmacological strategy $(10,47-49)$, followed by concurrent and/or strength training (50-52). Water exercise as a strategy for hypertension treatment is now better understood (46) and our study can provide valuable input on the post-exercise safety of this exercise modality.

\section{Study limitations}

The main limitation of this study is the conduction in a nonlaboratory setting making it difficult to control some environmental variables. However, it is important to mention that the study was performed under the same conditions as found in regular gyms and aquatic centers attended by this elderly population and in a scenario that is very close to that of the daily work of therapists and physical educators and trainers.

\section{Conclusion}

A significant increase in blood pressure was found immediately after a water aerobics session but it was a safe rise of small magnitude. BP returned to baseline within 10-20 minutes and remained stable until 30 minutes after exercise. These data further support the safe prescription of this form of exercise to hypertensive patients in general. Water aerobic exercise for hypertensive elderly people can help improve physical fitness and provide greater independence and autonomy, reduced risk of falls, and other benefits of water exercise.

\section{Acknowledgment}

The authors thank Carla Finger for her review of the English language and thank Coordination for the Improvement of Higher Education Personnel (CAPES) by the Raphael M Cunha, process n. BEX 8048/14-2.

\section{Declaration of interest}

The authors report no conflicts of interest.

\section{References}

1. Chobanian AV, Bakris GL, Black HR, et al. The seventh report of the joint national committee on prevention, detection, evaluation, and treatment of high blood pressure: the JNC 7 report. JAMA 2003;289(19):2560-72.

2. Picon RV, Fuchs FD, Moreira LB, Fuchs SC. Prevalence of hypertension among elderly persons in urban Brazil: a systematic review with meta-analysis. Am J Hypertens 2013;26 (4):541-8.

3. Safar ME, Benetos A. Factors influencing arterial stiffness in systolic hypertension in the elderly: role of sodium and the renin-angiotensin system. Am J Hypertens 2003;16(3):249-58.

4. Zieman SJ, Melenovsky V, Kass DA. Mechanisms, pathophysiology, and therapy of arterial stiffness. Arterioscler Thromb Vasc Biol 2005;25(5):932-43.

5. Aronow WS, Fleg JL, Pepine CJ, et al. ACCF/AHA 2011 expert consensus document on hypertension in the elderly: a report of the American College of Cardiology Foundation task force on clinical expert consensus documents developed in collaboration with the American Academy of Neurology, American Geriatrics Society, American Society for Preventive Cardiology, American Society of Hypertension, American Society of Nephrology, Association of Black Cardiologists, and European Society of Hypertension. J Am Soc Hypertens 2011;5(4):259-352.

6. Ogihara T, Rakugi H. Hypertension in the elderly: a Japanese perspective. Drugs Aging 2005;22(4):297-314.

7. Kim KI, Chang HJ, Cho YS, et al. Current status and characteristics of hypertension control in community resident elderly Korean people: data from a Korean longitudinal study on health and aging (KLoSHa study). Hypertens Res 2008;31 (1):97-105.

8. Macedo ME, Lima MJ, Silva AO, et al. Prevalence, awareness, treatment and control of hypertension in Portugal: the PAP study. J Hypertens 2005;23(9):1661-6.

9. Fuchs F, Gus M, Moreira W, et al. Blood pressure effects of antihypertensive drugs and changes in lifestyle in a Brazilian hypertensive cohort. J Hypertens 1997;15(7):783-92.

10. Cornelissen VA, Smart NA. Exercise training for blood pressure: a systematic review and meta-analysis. J Am Heart Assoc 2013;2(1): e004473.

11. Cunha RM, Jardim PC. Subacute blood pressure behavior in elderly hypertensive women after resistance exercise session. J Sports Med Phys Fitness 2012;52(2):175-80.

12. Floras JS, Sinkey CA, Aylward PE, et al. Postexercise hypotension and sympathoinhibition in borderline hypertensive men. Hypertension 1989;14(1):28-35.

13. Kiyonaga A, Arakawa K, Tanaka H, Shindo M. Blood pressure and hormonal responses to aerobic exercise. Hypertension 1985;7 (1):125-31.

14. Seals DR, Reiling MJ. Effect of regular exercise on 24-hour arterial pressure in older hypertensive humans. Hypertension 1991;18 (5):583-92.

15. Chen C, Bonham A. Postexercise hypotension: central mechanisms. Exerc Sport Sci Rev 2010;38(3):122-7.

16. Luttrell MJ, Halliwill JR. Recovery from exercise: vulnerable state, window of opportunity, or crystal ball? Front Physiol 2015;6:204

17. Santana HA, Moreira SR, Asano RY, et al. Exercise intensity modulates nitric oxide and blood pressure responses in hypertensive older women. Aging Clin Exp Res 2013;25(1):43-8.

18. Bocalini DS, Serra AJ, Murad N, Levy RF. Water- versus landbased exercise effects on physical fitness in older women. Geriatr Gerontol Int 2008;8(4):265-71.

19. Gappmaier E, Lake W, Nelson AG, Fisher AG. Aerobic exercise in water versus walking on land: effects on indices of fat reduction 
and weight loss of obese women. J Sports Med Phys Fitness 2006;46(4):564-9.

20. Barbosa TM, Garrido MF, Bragada J. Physiological adaptations to head-out aquatic exercises with different levels of body immersion. J Strength Cond Res 2007;21(4):1255-9.

21. Dehkordi AS, Sokhangeoi Y, MA. A. Impact of an aquatic exercising program on the balance of elderly males. Int Res J Appl Basic Sci 2012;21(4):1678-82.

22. Santos NS, Costa RFd, Kruel LFM. Effects of aquatic aerobic exercises on blood pressure in hypertensive adults: systematic review. Braz J Phys Act Health 2014;19(5):548-58.

23. Cider A, Sunnerhagen KS, Schaufelberger M, Andersson B. Cardiorespiratory effects of warm water immersion in elderly patients with chronic heart failure. Clin Physiol Funct Imaging 2005;25(6):313-7.

24. Park KS, Choi JK, Park YS. Cardiovascular regulation during water immersion. Appl Hum Sci 1999;18(6):233-41.

25. Hall J, Bisson D, O'Hare P. The physiology of immersion. Physiotherapy 1990;76:517-21.

26. Larochelle P, Cusson JR, du Souich P, et al. Renal effects of immersion in essential hypertension. Carvedilol Study Group. Am J Hypertens 1994;7(2):120-8.

27. Darby LA, Yaekle BC. Physiological responses during two types of exercise performed on land and in the water. J Sports Med Phys Fitness 2000;40(4):303-11.

28. Schulz KF, Altman DG, Moher D. CONSORT 2010 statement: updated guidelines for reporting parallel group randomised trials. BMJ 2010;340:c332.

29. Cunha RM, Macedo CB, Araujo SF, et al. Subacute blood pressure response in elderly hypertensive women after a water exercise session: a controlled clinical trial. High Blood Press Cardiovasc Prev 2012;19(4):223-7.

30. Kruel LF, Peyré-Tartaruga LA, Coertjens M, et al. Using heart rate to prescribe physical exercise during head-out water immersion. J Strength Conditioning Res 2014;28(1):281-9.

31. Cohen J. Statistical power analysis for the behavioral sciences. 2nd ed. New Jersey: Lawrence Erlbaum; 1988.

32. Lemes IR, Ferreira PH, Linares $\mathrm{SN}$, et al. Resistance training reduces systolic blood pressure in metabolic syndrome: a systematic review and meta-analysis of randomised controlled trials. Br J Sports Med. 2016 Mar 8. pii: bjsports-2015-094715. doi:10.1136/ bjsports-2015-094715. [Epub ahead of print]

33. Farahani AV, Mansournia MA, Asheri H, et al. The effects of a 10week water aerobic exercise on the resting blood pressure in patients with essential hypertension. Asian J Sports Med 1(3):159-67.

34. Yamazaki F, Sone R. Thermal stress modulates arterial pressure variability and arterial baroreflex response of heart rate during head-up tilt in humans. Eur J Appl Physiol 2001;84(4):350-7.

35. Kenney WL, Munce TA. Invited review: aging and human temperature regulation. J Appl Physiol (1985) 2003;95(6):2598-603.

36. Omvik P, Lund-Johansen P. Comparison of the hemodynamic effects of five calcium channel blockers at rest and during exercise in essential hypertension. Ann Clin Res 1988;20 Suppl 48:23-30.
37. Quigg R, Salyer J, Mohanty PK, Simpson P. Impaired exercise capacity late after cardiac transplantation: influence of chronotropic incompetence, hypertension, and calcium channel blockers. Am Heart J 1998;136(3):465-73.

38. Melo CM, Alencar Filho AC, Tinucci T, et al. Postexercise hypotension induced by low-intensity resistance exercise in hypertensive women receiving captopril. Blood Press Monit 2006;11 (4):183-9.

39. Lowenthal D, Powers S, Pollock M. Interactions of B-blockade and exercise: Implications and applications for the elderly. Am J Geri Card 1992;1:42-57.

40. Stein DT, Lowenthal DT, Porter RS, et al. Effects of nifedipine and verapamil on isometric and dynamic exercise in normal subjects. Am J Cardiol 1984;54(3):386-9.

41. Beevers G, Lip GY, O'Brien E. ABC of hypertension: the pathophysiology of hypertension. Br Med J 2001;322(7291):912.

42. Heithold K, Glass S. Variations in heart rate and perception of effort during land and water aerobics in older women. J Exercise Physiol 2002;4:22-8.

43. Neves EB, Moreira TR, Lemos R, et al. Using skin temperature and muscle thickness to assess muscle response to strength training. Rev Bras Med Esporte 2015;21:350-4.

44. Craig AB, Jr., Dvorak M. Thermal regulation during water immersion. J Appl Physiol 1966;21(5):1577-85.

45. McArdle WD, Toner MM, Magel JR, et al. Thermal responses of men and women during cold-water immersion: influence of exercise intensity. Eur J Appl Physiol Occup Physiol 1992;65 (3):265-70.

46. Terblanche E, Millen AM. The magnitude and duration of postexercise hypotension after land and water exercises. Eur J Appl Physiol 2012;112(12):4111-8.

47. Pescatello LS, MacDonald HV, Lamberti L, Johnson BT. Exercise for hypertension: a prescription update integrating existing recommendations with emerging research. Curr Hypertens Rep 2015;17(11):87.

48. Ciolac EG, Guimaraes GV, D'Avila VM, et al. Acute aerobic exercise reduces 24-h ambulatory blood pressure levels in longterm-treated hypertensive patients. Clinics (Sao Paulo) 2008;63 (6):753-8.

49. Whelton SP, Chin A, Xin X, He J. Effect of aerobic exercise on blood pressure: a meta-analysis of randomized, controlled trials. Ann Int Med 2002;136(7):493-503.

50. Cornelissen VA, Fagard RH, Coeckelberghs E, Vanhees L. Impact of resistance training on blood pressure and other cardiovascular risk factors: a meta-analysis of randomized, controlled trials. Hypertension 2011;58(5):950-8.

51. Keese F, Farinatti P, Pescatello L, Monteiro W. A comparison of the immediate effects of resistance, aerobic, and concurrent exercise on postexercise hypotension. J Strength Cond Res 2011;25 (5):1429-36.

52. Teixeira L, Ritti-Dias RM, Tinucci T, et al. Post-concurrent exercise hemodynamics and cardiac autonomic modulation. Eur J Appl Physiol 2011;111(9):2069-78. 